\title{
AVALIAÇÃO DA FORÇA MUSCULAR DE MEMBROS INFERIORES EM IDOSAS PRATICANTES DE ATIVIDADE FÍSICA
}

Giovanna Neves Bresqui ${ }^{1}$, Elaine Aparecida Lozano da Silva ${ }^{1}$, Juliana Rosini da Silva ${ }^{2}$, Vinícius Gustavo Gimenes Turato ${ }^{2}$, Andressa Sampaio Pereira ${ }^{3}$, Cláudia Regina Sgobbi de Faria ${ }^{4}$, Regina Celi Trindade Camargo ${ }^{4}$

Universidade Estadual Paulista - FCT/UNESP. ${ }^{1}$ Programa de Pós Graduação Lato Sensu - Especialização em Fisioterapia aplicada a Gerontologia. ${ }^{2}$ Programa de Residência em Saúde, área de Reabilitação Física. ${ }^{3}$ Curso de Fisioterapia, ${ }^{4}$ Departamento de Fisioterapia, Presidente Prudente, SP. e-mail: giovannanb_92@hotmail.com

\section{RESUMO}

O envelhecimento é acompanhado de várias manifestações fisiológicas, dentre elas, a redução da força muscular, sendo considerado um dos fatores determinantes para a manutenção da capacidade funcional, pois está relacionada diretamente com a independência e a realização das atividades de vida diária, assim como a qualidade de vida dos idosos. O objetivo do estudo foi analisar a força muscular de membros inferiores em idosas praticantes de atividade física. Trata-se de uma amostra composta por 62 voluntários do sexo feminino com idade igual ou superior a 60 anos. Realizou-se o teste de uma repetição máxima (1RM). Os resultados demonstraram aumento significativo da força muscular em relação à extensão e flexão de joelho comparando-se os períodos de pré e pós-teste. Portanto, conclui-se que a prática de atividade física favorece o aumento da força muscular de membros inferiores, que contribui com a melhora da qualidade de vida dos idosos.

Palavras-chave: atividade física, envelhecimento, força muscular, qualidade de vida, idosos.

\section{EVALUATION OF MUSCLE STRENGTH OF LOWER LIMBS IN ELDERLY PRACTITIONERS OF PHYSICAL ACTIVITY}

\begin{abstract}
Aging is accompanied by various physiological manifestations, among them, reduced muscle strength, being considered one of the determinants for the maintenance of functional capacity factors, because it is directly related to the independence and performance of daily living activities, as well as quality of life for seniors. The aim of the study was to analyze the muscle strength of the lower limbs in elderly women engaged in physical activity. This is a sample of 62 female volunteers aged over 60 years. We carried out the one-repetition maximum (1RM) test. The results demonstrated a significant increase in muscle strength in relation to the extension and knee flexion comparing the periods pre and post-test. Therefore, it is concluded that physical activity promotes increased muscle strength of the lower limbs, which contributes to improved quality of life for seniors.
\end{abstract}

Keywords: physical activity, aging, muscle strength, quality of life, the elderly. 


\section{INTRODUÇÃO}

Atualmente, o processo de envelhecimento vem apresentando um crescimento acelerado em contexto mundial, justificado por meio da redução das taxas de natalidade e mortalidade, assim como o aumento da expectativa de vida. De acordo com a Organização Mundial da Saúde (OMS), estima-se que em 2025, o Brasil será o sexto país com o maior contingente de idosos no mundo, de tal forma que ultrapasse 30 milhões de pessoas, isso ocorre devido ao aumento da expectativa de vida. ${ }^{1,2}$

Em 2009, o Instituto Brasileiro de Geografia e Estatística (IBGE) verificou um aumento de 697 mil pessoas com 60 anos ou mais, representando um crescimento de $3,3 \%$ na população de idosos. Dentre esses idosos, a maioria é acompanhada por doenças crônicas e limitações funcionais. ${ }^{3}$

O envelhecimento é acompanhado de várias manifestações fisiológicas, dentre elas, a redução da força muscular, que vem a diminuir, a cada década, em torno de $15 \%$ a partir dos 50 anos e aos 70 anos ocorre perda em média de $30 \%$ por década. A força muscular é um dos fatores determinantes para a manutenção da capacidade funcional, pois está relacionada diretamente com a independência e a realização das atividades de vida diária (AVD's), assim como a qualidade de vida dos idosos. ${ }^{4,5}$
A força máxima do indivíduo é descrita como a tensão gerada contra uma resistência por meio da capacidade máxima de um músculo ou grupo muscular. Essa pode ser medida através do teste de uma repetição máxima (1RM), definido como " $a$ maior carga que pode ser movida por uma amplitude específica de movimento uma única vez, sem compensações musculares $e$ de forma correta". 6

Com o avançar da idade, além da diminuição de força, verifica-se uma redução da potência muscular, decorrente de uma predominante perda das fibras do tipo II, que limita a capacidade de gerar força rapidamente. ${ }^{7}$ A redução de força e potência muscular, além de serem limitantes para a funcionalidade do idoso, aumenta o risco de quedas e gera déficits de equilíbrio. ${ }^{4}$

De acordo com isso, várias pesquisas mostram que a prática regular de atividade física na terceira idade é capaz de minimizar ou prevenir os efeitos deletérios do envelhecimento, atuando diretamente no aumento da expectativa de vida do idoso por meio dos seus benefícios. Alguns desses benefícios são: aumento de potência muscular e força em torno de $20 \%$ a $30 \%$, ganho de massa muscular e óssea, aumento da velocidade de contratilidade das fibras do tipo I e II, melhora da funcionalidade e capacidade de manter a realização das AVD's. $^{8,7}$ 
Portanto, a prática de atividade física é de extrema importância para a manutenção da força muscular do idoso, assim como a preservação da saúde e qualidade de vida desses indivíduos. ${ }^{7}$

O objetivo do estudo foi analisar os efeitos do exercício físico sobre a força muscular de membros inferiores em mulheres com idade igual ou superior a 60 anos que participam de um programa de fisioterapia caracterizado por exercícios físicos regulares e supervisionados.

\section{MÉTODOS}

Trata-se de uma amostra composta por 62 voluntários do sexo feminino, participantes de programa de fisioterapia preventiva para a terceira idade em Presidente Prudente, realizado de duas a três vezes na semana, com duração de uma hora.

Os critérios de inclusão do presente estudo foram: idosas do sexo feminino com idade igual ou superior à 60 anos, participantes do programa de fisioterapia preventiva para a terceira idade da FCT/UNESP, estabilidade clínica com apresentação de atestado médico constatando que as mesmas estão aptas a realizarem atividade física de moderada intensidade.

Os critérios de exclusão foram: idosos do sexo masculino, pneumopatas, cardiopatas, indivíduos que tenham realizando algum procedimento cirúrgico recentemente ou que apresentaram algum tipo de intercorrência durante as avaliações.

As voluntárias assinaram um Termo de Consentimento Livre e Esclarecido, após ficarem cientes dos objetivos e procedimentos dessa pesquisa.

O projeto foi encaminhado ao Comitê de Ética em Pesquisa (CEP) da Faculdade de Ciências e Tecnologia - UNESP, Campus de Presidente Prudente-SP, com número de protocolo: 6654813.0.0000.5402.

\section{Protocolo de Avaliação}

As pacientes foram divididas em dois grupos de acordo com os dias em que frequentavam o programa de fisioterapia. 0 programa realizado duas vezes na semana corresponde ao P2 $(n=34)$ e o de três vezes na semana refere-se ao P3 $(n=28)$.

Realizou-se uma avaliação inicial no qual foi coletada a força muscular de membros inferiores (MMII) em um aparelho de musculação. Após a coleta, as pacientes realizaram o protocolo de Exercícios Físicos Supervisionados (EFS) em um período de seis meses e suas atividades rotineiras realizadas normalmente.

Após esse período, efetuou-se uma avaliação final com a mesma coleta de força muscular realizada na avaliação inicial. 


\section{Procedimento de Coleta de Dados}

Teste de uma repetição máxima (1RM)

Realizou-se esse teste para avaliar a força máxima de MMII, determinando o valor de uma repetição máxima (1RM) em um aparelho de musculação. Foram testados os seguintes grupos musculares: Músculo semitendíneo, semimembranáceo e bíceps femoral, relacionados à flexão de joelho. E músculo quadríceps, responsável pela extensão de joelho.

Para o exercício resistido do teste foi estipulada uma carga inicial de $20 \%$ do peso corporal para os membros inferiores. Antes de iniciar o teste foram verificados os sinais vitais, como: pressão arterial (PA), frequência cardíaca (FC), saturação de oxigênio ( $\mathrm{SpO} 2$ ) e escala de Borg. Após o descanso, realizou-se o movimento com a nova carga acrescentada.

Concluía-se o teste quando a voluntária atingisse a carga que provocasse falha mecânica durante a execução do movimento e estabelecia como carga máxima a última carga que conseguiu realizar o exercício sem falha mecânica. Não foram feitas mais do que cinco tentativas para se estabelecer a carga máxima do teste. Seria considerado inválido se necessitasse de mais tentativas, assim a voluntária seria submetida à realização do teste em outro dia. ${ }^{9}$

\section{Protocolo de exercícios}

As voluntárias participaram de um programa de fisioterapia preventiva por um período de seis meses, com duração de uma hora e supervisão adequada.

Eram verificados os sinais vitais no início e término das sessões (cinco minutos antes e após): PA e FC, com o intuito de minimizar riscos de intercorrências cardiovasculares durante os exercícios.

As aulas eram compostas de alongamentos específicos e gerais, exercícios de aquecimento, fortalecimento muscular, coordenação motora, equilíbrio, flexibilidade e exercícios de relaxamento ao final do programa.

\section{Análise Estatística}

Para análise dos dados foi utilizado o programa estatístico GraphPadPrism ${ }^{\circledR}$ 5.0. A normalidade na distribuição dos dados foi avaliada por meio do teste de Shapiro-Wilk. Aplicou-se o teste $\mathrm{t}$ Student ou teste de Mann-Whitney conforme a normalidade dos dados para a análise intergrupos (Grupo P2 versus Grupo P3) e intragrupos (antes e após - programa de exercícios). Para a comparação de dados categóricos (questionário QUASI) foi utilizado o teste de Wilcoxon e, as demais variáveis do estudo descritas por análise de frequência e porcentagem (dados socioeconômicos e GDS). O nível de significância utilizado foi de $p<0,05$ 


\section{RESULTADOS}

Os resultados foram descritos de forma qualitativa e quantitativa. Para esta, realizou-se a análise estatística, utilizando o programa Statística (StatSoft. Inc., 1991).

Os dados foram expressos em média \pm desvio-padrão (DP) e encontram-se dispostos em formato de tabela.

A tabela 1 demonstra as comparações intragrupos (P2 e P3) da avaliação de força por meio do teste de 1RM. Observou-se que - P2 obteve aumento significativo da força muscular na extensão de joelho comparando- se o pré e pós-teste. O P3 apresentou ganhos significativos na flexão, porém para a extensão de joelho foram extremamente significativos em relação em pré e pós-teste.

Tabela 1. Valores de média \pm desvio-padrão (DP) dos grupos no teste de 1RM.

\begin{tabular}{l|l|l|l}
\hline & INICIAL & FINAL & Valor de $p$ \\
\hline P2 & & & \\
\hline FLEXÃO & $22,65 \pm 5,67$ & $23,82 \pm 6,97$ & 0,3014 \\
\hline EXTENSÃO & $30,88 \pm 7,54$ & $36,18 \pm 8,17$ & $0,0024^{* *}$ \\
\hline
\end{tabular}

\begin{tabular}{l|l|l|l}
\hline P3 & & & \\
\hline FLEXÃO & $23,21 \pm 7,23$ & $26,79 \pm 6,12$ & $0,0045^{* *}$ \\
\hline EXTENSÃO & $31,43 \pm 8,48$ & $38,21 \pm 8,19$ & $<0,001^{* * *}$ \\
\hline
\end{tabular}

A análise dos resultados de intragrupos no teste de $1 R M$ identificou diferença $(\Delta)$ entre os valores iniciais e finais para os movimentos em flexão e extensão de joelho e, em seguida, por meio de testes de correlação esses valores foram relacionados com os grupos. Porém, nesta comparação, não houve diferença significativa $(p>0,05)$. De acordo com isso, verifica-se que não há interferência nos resultados do teste para flexão e extensão de joelho se o indivíduo participa duas ou três vezes na semana, ou seja, a frequência no programa não interfere diretamente no teste. 


\section{DISCUSSÃO}

No presente estudo, realizou-se um programa de fisioterapia preventiva em idosas durante 24 semanas contendo um protocolo de exercícios com pesos (halteres e caneleiras). Após esse período, através do teste de 1RM, ambos os grupos apresentaram ganhos significativos na força muscular de membros inferiores, tanto para extensão quanto para flexão de joelho.

Esses dados corroboram com 0 estudo de Silva et $a^{10}$, que submeteram um treinamento de força para 30 mulheres idosas com idade acima de 50 anos por um período de 12 semanas, sendo três sessões semanais. Após o treino, a força muscular máxima foi avaliada por meio do teste de $1 R M$, no qual os autores concluíram que apesar de não haver alterações na gordura corporal e na massa magra, observaram aumentos significativos na força muscular para os movimentos de flexão e extensão de joelho.

Assim como no estudo de Santos et al $^{11}$, que avaliou a força muscular de 52 mulheres idosas com idade acima de 60 anos. Foram distribuídas em Grupo Experimental (GE) e Grupo Controle (GC). O GE realizou 12 semanas de tratamento com protocolo de exercícios resistidos e após esse período, ambos os grupos foram submetidos ao teste de 1RM. Houve aumento de força muscular no GE, comparando-se o período de pré e pós-teste. E também se mostrou com maiores níveis quando relacionado ao GC, que não realizou o treinamento de força.

Como foi visto, o processo de envelhecimento é acompanhado pela perda progressiva de força e massa muscular que se associam com a atrofia e perda de fibras musculares. Estudos apontam que após a quinta década de vida, as mulheres apresentam maior perda de massa muscular. Isso se deve por ser um período que normalmente se inicia o processo de menopausa, o que ressalta o papel dos hormônios ovarianos na regulação da massa muscular. Portanto, existe uma relação direta entre a perda de força muscular e a diminuição da massa em mulheres com idade acima de 50 anos. $^{9}$

A perda de força muscular nos idosos também é acompanhada pela diminuição da independência funcional, principalmente em atividades que exigem a força dos MMII, como caminhar, subir e descer escadas, sentar e levantar. Isso se deve à fraqueza muscular, que predomina mais em MMII do que nos membros superiores (MMSS). ${ }^{9}$

Diversos estudos ${ }^{7,9,11}$ demonstram que a melhor opção para prevenir ou minimizar a perda de força em idosos é a prática de atividade física, sobretudo quando se utiliza protocolos com exercícios resistidos, pois além de atuar de maneira significativa na força e massa muscular, 
melhora a qualidade de vida, o estado de saúde, mantém a independência funcional, minimizando as alterações decorrentes do envelhecimento.

\section{CONCLUSÃO}

De acordo com esse estudo, podemos observar que ao avaliarmos o efeito do exercício físico na força de membros inferiores em mulheres idosas, inseridas em um programa de fisioterapia preventiva, encontramos grandes benefícios e ganhos significativos na força muscular de membros inferiores, podendo assim prevenir ou minimizar as alterações decorrentes do envelhecimento, além de melhorar a qualidade de vida e a independência funcional dessas idosas.

\section{REFERÊNCIAS}

1. Maués CR, Paschoal SMP, Jaluul $O$, França CC, Filho WJ. Avaliação da qualidade de vida: comparação entre idosos jovens e muito idosos. Rev Bras Clin Med. 2010; 8(5):405-410.

2. Silva MF, Goulart NBA, Lanferdini FJ, Marcon $M$, Dias CP. Relação entre os níveis de atividade física e qualidade de vida de idosos sedentários e fisicamente ativos. Rev Bras Geriatr e Gerontol. 2012; 15(4):635-642. http://dx.doi.org/10.1590/s1809$\underline{98232012000400004}$

3. Vilela DS, Busanello KLS, Oliveira SD, Fréz AR, Riedi C. Correlação entre o estado geral de saúde e a capacidade funcional de idosos ativos. Cons Saúde. 2013; 12(3):447454.
4. Wibelinger LM, Schneider $\mathrm{RH}$, Tonial A, Oliveira G, Klein B, Capitânio D. Avaliação da força muscular de flexores e extensores de joelho em indivíduos idosos socialmente ativos. Rev Bras Cienc Envelhecim Hum. 2009; 6(2):284-292. http://dx.doi.org/10.5335/rbceh.2009.02 $\underline{7}$

5. Pereira FD, Batista WO, Furtado HL, Junior EDA, Giani TS, Dantas EHM. Comparação da força funcional de membros inferiores e superiores entre idosas fisicamente ativas e sedentárias. Rev Bras Geriatr e Gerontol. 2009; 12(3):417-427.

6. Cardoso FS, Curtolo M, Natour J, Junior IL. Avaliação da qualidade de vida, força muscular e capacidade funcional em mulheres com fibromialgia. Rev Bras Reumatol. 2011; 51(4):338-350. http://dx.doi.org/10.1590/S0482$\underline{50042011000400006}$

7. Rocha $A C$, Fernandes MC, Dubas JP, Júnior DPG. Análise comparativa da força muscular entre idosas praticantes de musculação, ginástica localizada e institucionalizada. Fit Perf J. 2009; 8(1):16-20.

8. Gobbi S, Corazza DI, Costa JRL, Ueno DT, Gobbi LTB. Atividade física e saúde no envelhecimento: a experiência do Programa de Atividade Física para a Terceira Idade (PROFIT). In: Rosa TEC, Barroso ÁES, Louvison MCP. Velhices: experiências e desafios nas políticas do envelhecimento ativo. São Paulo. 2013 p. 283-297.

9. Orsatti $\mathrm{FL}$, Dalanesi RC, Maestá $\mathrm{N}$, Náhas EAP, Burini RC. Redução da força muscular está relacionada à perda muscular em mulheres acima de 40 anos. Rev Bras Cineantropom Desempenho Hum. 2011; 13(1):36-42.

10. Silva $C M$, Gurjão $A L D$, Ferreira $L$, Gobbi LTB, Gobbi S. Efeito do treinamento 
com pesos, prescrito por zona de repetições máximas, na força muscular e composição corporal em idosas. Rev Bras Cineantropom Desempenho Hum. 2006; 8(4):39-45.

11. Santos LTA, Vale RGS, Melo DB, Giani TS, Dantas EHM. Efeitos da cinesioterapia sobre os níveis de IGF-1, força muscular e autonomia funcional em mulheres idosas. Rev Bras Cineantropom Desempenho Hum. 2010; 12(6):451-456.

http://dx.doi.org/10.5007/1980-

$\underline{0037.2010 v 12 n 6 p 451}$

Recebido para publicação em 12/08/2014

Revisado em 21/01/2015

Aceito em 16/04/2015 